\title{
Simulação clínica no desempenho cognitivo, satisfação e autoconfiança na aprendizagem: estudo quase-experimental
}

Clinical simulation in cognitive performance, satisfaction and self-

confidence in learning: a quasi-experimental study

Simulacro clínico en el rendimiento cognitivo, satisfacción y autoconfianza

en el aprendizaje: estudio cuasi experimental

Raphael Raniere de Oliveira Costa ${ }^{1}$ id https://orcid.org/0000-0002-4199-9696

Soraya Maria de Medeiros ${ }^{1}$ if https://oridid.org/0000-0002-6626-5963

Verónica Rita Dias Coutinho io https://orcid.org/0000-0001-8073-4562

Cristina Maria Figueira Veríssimo² io https://orcid.org/0000-0002-8836-2828

Margarida Alexandra Nunes Carramanho Gomes Martins Moreira da Silva io hitps://orcid.org/0000-0003-0031-271X

Eudes Euler de Souza Lucena ${ }^{1}$ io hitps://orcid.org/0000-0003-3119-7822

Como citar:

Costa RR, Medeiros SM, Coutinho VR, Veríssimo CM, Silva MA, Lucena EE, et al. Simulação clínica no desempenho cognitivo, satisfação e autoconfiança na aprendizagem: estudo quase-experimental. Acta Paul Enferm. 2020;33:eAPE20180123.

DOI

http://dx.doi.org/10.37689/acta-

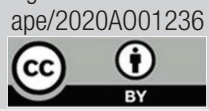

Descritores

magem; Atenção primária à saúde

Keywords

Simulation; Education, nursing; Primary health care

Descriptores

Simulación; Educación en enfermería; Atenção

primária à saúde

Submetido 16 de Maio de 2019

Aceito

10 de Março de 2020

Autor correspondente

Raphael Raniere de Oliveira Costa

E-mail: raphaelraniere@hotmail.com

\section{Resumo}

Objetivo: Analisar o conhecimento cognitivo, a satisfação e a autoconfiança de estudantes de enfermagem a partir do uso da simulação no ensino de consulta de enfermagem em vacinação no contexto da enfermagem comunitária.

Métodos: Estudo de intervenção, quantitativo, compreendido em duas fases. Foi utilizado o delineamento quase-experimental de grupo controle não equivalente do tipo pré-teste e pós-teste. Foram respeitados todos os aspectos éticos. A amostra final foi composta por 94 estudantes de enfermagem portugueses. Os estudantes foram alocados em dois grupos: controle e experimental. 0 grupo controle teve acesso a uma aula de habilidade tradicional. 0 grupo experimental participou de uma sessão de simulação com dois casos. Além da caracterização sociodemográfica, os estudantes responderam a um pré-teste e pós-teste para aferir o desempenho cognitivo. Além disso, foi aplicada uma escala de satisfação e autoconfiança na aprendizagem. Adotou-se um nível de significância de $5 \%$.

Resultados: Dos 94 estudantes, (95,7\%) são do sexo feminino, com faixa etária entre 18 e 20 anos (91,5\%), solteiros $(98,9 \%)$, sem vínculo laboral (94,7\%). A partir da simulação clínica os estudantes aprendem mais quando comparado aos estudantes que participaram da estratégia tradicional ( $p$ valor $=0,000)$. Além disso, a simulação clínica promove satisfação entre os estudantes.

Conclusão: Por ser uma estratégia de ensino e aprendizagem que permite um melhor desempenho cognitivo, por gerar satisfação nos estudantes, a simulação clínica pode ser apontada como uma estratégia potencial para 0 ensino em enfermagem comunitária.

\section{Abstract}

Objective: To analyze the cognitive knowledge, satisfaction and self-confidence of nursing students based on simulation use in teaching nursing consultation in community nursing vaccination.

Methods: An intervention and quantitative study comprised in two phases. A quasi-experimental design of a control group not equivalent to the pre- and post-test type was used. All ethical aspects have been respected. The final sample consisted of 94 Portuguese nursing students. They were allocated into two groups: control and experimental. The control group had access to a traditional skill class. The experimental group participated in a simulation session with two cases. In addition to the sociodemographic characterization, the students answered a pre- and post-test to measure cognitive performance. Moreover, satisfaction and self-confidence in learning scale was applied. A significance level of $5 \%$ was adopted.

Results: Of the 94 students, 95.7\% were female, 91.5\% were aged between 18 and 20 years, 98.9\% were single and $94.7 \%$ were unemployed. From the clinical simulation, students learn more when compared to 
students who participated in the traditional strategy $(p$ value $=0.000$ ). Furthermore, clinical simulation promotes satisfaction among students.

Conclusion: Since it is a teaching and learning strategy that allows a better cognitive performance, and because it generates satisfaction among students, clinical simulation can be pointed out as a potential strategy for teaching in community nursing.

Universal Trial Number: U1111-1227-2241

\section{Resumen}

Objetivo: Analizar el conocimiento cognitivo, la satisfacción y la autoconfianza de estudiantes de enfermería a partir del uso de simulacro en la enseñanza de consulta de enfermería en vacunación, en el contexto de enfermería comunitaria.

Métodos: Estudio experimental, cuantitativo, realizado en dos fases. Se utilizó el diseño cuasi experimental de grupo de control no equivalente tipo pretest y postest. Se respetaron todos los aspectos éticos. La muestra final estuvo compuesta por 94 estudiantes de enfermería portugueses. Los estudiantes fueron separados en dos grupos: control y experimental. El grupo de control tuvo acceso a una clase de habilidad tradicional. El grupo experimental participó en una sesión de simulacro con dos casos. Además de la caracterización sociodemográfica, los estudiantes respondieron un pretest y postest para determinar el rendimiento cognitivo. También se aplicó una escala de satisfacción y autoconfianza en el aprendizaje. Se adoptó un nivel de significación de 5 \%.

Resultados: De los 94 estudiantes, el 95,7 \% era de sexo femenino, del grupo de edad entre 18 y 20 años (91,5\%), solteros (98,9 \%), sin vínculo laboral $(94,7 \%)$. A partir del simulacro clínico, los estudiantes aprendieron más en comparación con los estudiantes que participaron de la estrategia tradicional ( $\mathrm{p}$ valor =0,000). Además, el simulacro clínico promueve la satisfacción de los estudiantes.

Conclusión: Por ser una estrategia de enseñanza y aprendizaje que permite un mejor rendimiento cognitivo, por generar satisfacción en los estudiantes, el simulacro clínico puede indicarse como una estrategia potencial para la enseñanza de enfermería comunitaria.

\section{Introdução}

Pensar no ensino de enfermagem no contexto do mundo atual do trabalho implica refletir sobre o investimento em estruturas e recursos humanos, na diversificação de estratégias de ensino e aprendizagem, na minimização dos erros em saúde e de enfermagem, na garantia da segurança do paciente, na qualificação da força de trabalho, no respeito e condição de saúde individual e coletiva, na integralidade e humanização da assistência, entre outras questôes. ${ }^{(1)}$

É importante destacar que o contato entre estudantes, profissionais e doentes/usuários deve ser mediado a partir do conhecimento, competências e habilidades, e que esta interação deve ser orientada por princípios bioéticos e dos direitos humanos. ${ }^{(1)}$ Nessa perspectiva, partilha-se do pensamento de que para esse profissional possa chegar mais preparado na prática real, é imprescindível que este vivencie, durante a formação, diferentes momentos que oportunize o desenvolvimento dessas destrezas.

Para tanto, pensa-se em estratégias de ensino e aprendizagem que sejam capazes de melhorar o desempenho cognitivo do estudante, que estimule estes a serem mais autoconfiantes e que promova satisfação. A simulação contempla essas perspectivas uma vez que promove a aprendizagem significativa e reproduz diferentes realidades em saúde. ${ }^{(2)}$
No ensino de enfermagem a simulaçáo é identificada como uma estratégia de ensino que utiliza tecnologias para replicar cenários que simulam a prática, em ambiente controlado e realista, onde o estudante participa ativamente do processo de ensino e aprendizagem com a finalidade de praticar exaustivamente, aprender, refletir e avaliar produtos e processos. ${ }^{(3)} \mathrm{Na}$ simulação, os elementos partícipes são: o facilitador; os participantes; práticas educacionais (caso clínico e a simulação clínica); características do desenho de simulação (com foco em resoluçáo de problemas) e expectativa dos resultados das simulaçôes (debriefing). ${ }^{(4)}$

Neste estudo, o conceito de conhecimento cognitivo utilizado parte da perspectiva teórica da Pirâmide de Miller. O "sabe", base da pirâmide, se refere a avaliação de como os estudantes integram os conhecimentos prévios com a nova informação: o saber. $\mathrm{O}$ conhecimento, na perspectiva teórica, é de fundamental relevância para que o estudante possa atingir outros níveis da pirâmide: o "sabe como", o "mostra como", e o "faz". (5)

A satisfação é entendida como um sentimento de prazer dada pelo alcance daquilo que se espera. ${ }^{(6)}$ No tocante à satisfaçáo do estudante, esta pode ser associada a maior envolvimento e motivação para aprender. Além disso, pode ser um indicador para avaliar as estratégias de ensino adotadas durante o percurso de formação dos estudantes com o objetivo de melhorar a qualidade do ensino. ${ }^{(7)}$ Já o conceito de autoconfiança está relacionado ao demonstrar 
crença no sucesso das açóes nas competências e habilidades cognitivas, psicomotoras e atitudinais. ${ }^{(4,7)}$

Em Portugal, a prática simulada faz parte do currículo de muitas escolas de enfermagem. ${ }^{(6)}$ Porém, na realidade estudada, o uso da simulaçáo para o aprendizado de alguns conteúdos de Enfermagem comunitária, como imunização, ainda tem sido pouco explorado. Logo, identificar a eficácia, a satisfação e autoconfiança dos estudantes a partir do uso dessa estratégia, torna-se um passo importante para a ampliação do uso da simulação na Escola Superior de Enfermagem de Coimbra (ESEnfC). Nessa perspectiva, o estudo teve por objetivo analisar o conhecimento cognitivo, a satisfação e a autoconfiança de estudantes de enfermagem a partir do uso da simulação no ensino de consulta de enfermagem em vacinaçáo no contexto da enfermagem comunitária.

\section{Métodos}

Trata-se de um estudo de intervenção, quantitativo, compreendido em duas fases. Foi utilizado o delineamento quase-experimental de grupo controle não equivalente do tipo pré-teste e pós-teste. ${ }^{(8)}$

Por se tratar de uma pesquisa envolvendo seres humanos, o estudo levou em consideração a garantia dos princípios éticos e legais que regem a pesquisa em seres humanos em Portugal. Foi submetido e aprovado junto ao Comitê de Ética em Pesquisa da Escola Superior de Enfermagem de Coimbra sob o parecer $n^{\circ}$ P454-09/2017 e Universal Trial Number TN U11111227-2241. Os estudantes foram informados quanto aos objetivos, da possibilidade de abandono a qualquer momento sem consequências adversas, do caráter confidencial das repostas e dos procedimentos que foram adotados durante a coleta de dados. Ainda foram informados que este estudo não tinha qualquer influência nas notas finais da unidade curricular.

O estudo foi realizado na Escola Superior de Enfermagem de Coimbra, Portugal, com estudantes do $2^{\circ}$ ano do Curso de Licenciatura em Enfermagem na Unidade Curricular de Enfermagem Comunitária e Familiar na componente pratica laboratorial. A amostra inicial, do tipo não probabilística por conveniência, foi de 120 estudantes. No segundo semestre de 2018, a unidade curricular foi ofertada em quatro turmas. Os estudantes foram alocados em dois grupos: Grupo Controle (GC) e Grupo Experimental (GE). É válido ressaltar que a alocação não obedeceu a critérios de méritos dos pesquisados, e se deu por questóes da disponibilidade de tempo do pesquisador e a agenda de trabalho da pesquisa.

Foram incluídos os estudantes da graduação em enfermagem regulamente matriculados na Unidade Curricular de Enfermagem Comunitária e Familiar que estiverem presentes durante a aplicação dos instrumentos da pesquisa, das intervençóes e que preencheram corretamente os instrumentos do estudo. Foram excluídos os estudantes que não estiveram presentes, na aula em que foram colhidos os dados, bem como aqueles que responderam parcialmente aos instrumentos do estudo. Após aplicação dos critérios de elegibilidade, a amostra final foi constituída por 94 estudantes - conforme apresentado no diagrama de seguimento (Figura 1).

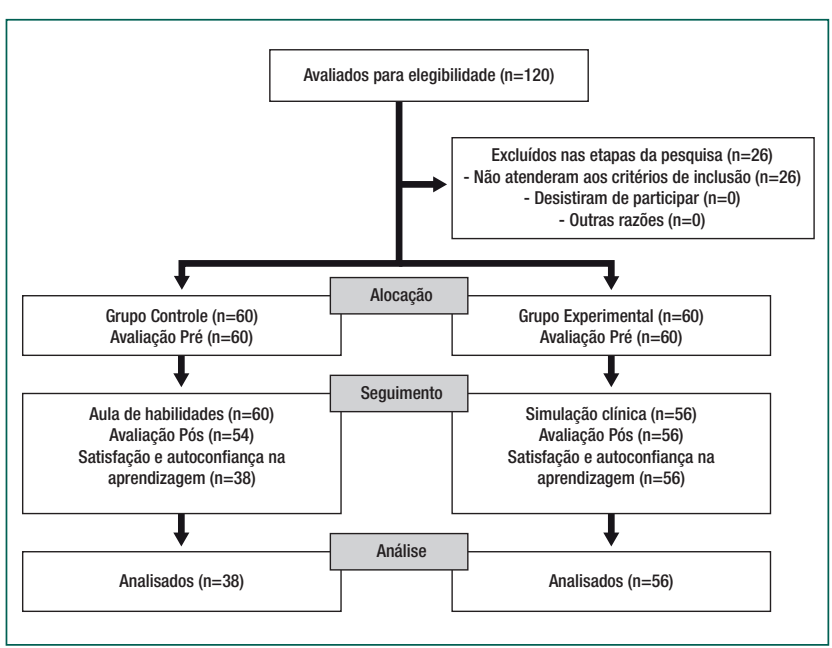

Figura 1. Diagrama de seguimento

Os estudantes do GC preencheram o questionário sociodemográfico, responderam ao teste de desempenho cognitivo e tiveram acesso ao ensino tradicional - uma aula de habilidades referente a imunização no contexto da enfermagem comunitária - e, no final, responderam novamente ao teste de desempenho, e preencheram a escala de satisfação e autoconfiança na aprendizagem.

A aula tradicional foi realizada a partir da exposição teórica em power point e a resolução de casos 
clínicos. No treino de habilidades, os estudantes treinaram técnicas de administração de vacinas.

Já os estudantes do GE, preencheram o questionário sociodemográfico, responderam ao teste de desempenho cognitivo e participaram de dois cenários de simulação com debriefing referente à consulta de enfermagem em vacinação no contexto da enfermagem comunitária e, no final, responderam novamente ao teste de desempenho cognitivo, e preencheram a escala de satisfação e autoconfiança na aprendizagem.

O questionário sociodemográfico possuía as seguintes variáveis: idade, sexo, estado civil, vínculo laboral e área de preferência da enfermagem. Quanto ao teste de desempenho cognitivo, as questôes expostas -total de cinco- contemplavam casos clínicos com situaçôes comumente encontradas nas consultas de enfermagem em vacinação em diferentes ciclos de vida. As questóes eram do tipo múltiplas escolhas de cinco itens. Destaca-se que o teste cognitivo foi desenvolvido pelos pesquisadores e tiveram o seu conteúdo validado pelos docentes da unidade curricular. Todos os envolvidos eram enfermeiros, com doutorado em enfermagem, e experiência profissional em simulação clínica. Para tanto, o teste foi apreciado por quatro docentes. Não houve discordância quanto ao conteúdo das questôes da avaliação. O valor global do teste era de 10,0 pontos, sendo 2,00 por questão.

$\mathrm{Na}$ sessão de simulação, foi seguido o modelo conceitual de Jeffries. ${ }^{(4)}$ Foram construídos dois cenários: um referente a um acidente traumático acidental doméstico (adulto) e um segundo relacionado a uma falsa contraindicação em criança. Os cenários também foram construídos e validados pela mesma equipe envolvida na construção do teste de desempenho cognitivo.

Nos cenários que foram simulados, utilizou-se a ferramenta paciente-padrão. Paciente-padrão são atores treinados para atuar e reproduzir comportamento de usuários em diversas situaçóes e estabelecimentos de assistência à saúde. ${ }^{(9)}$ Dois atores foram treinados previamente quanto à fala, reação e linguagem corporal.

O cenário 1, possuía os seguintes objetivos de aprendizagem: vivenciar situaçôes de manejo de cri- se ao cliente acometido por evento traumático/acidental no contexto da Unidade de Saúde Familiar; e propor a propedêutica mais adequada de manejo ao cliente acometido por evento traumático/acidental sem comprovação de situação vacinal. Neste cenário, utilizou-se moulage para caracterizar o paciente. Foi reproduzida uma lesão que caracterizasse um acidente com tesoura para o corte de uvas. A duração total da sessão foi de 45 minutos (15 para a execução do caso e 30 para o debriefing).

No cenário 2, os objetivos da aprendizagem eram os seguintes: vivenciar situaçóes de manejo de situações de recusa na administração de imunobiológicos em criança no contexto da Unidade de Saúde Familiar; averiguar a situação vacinal da criança; reconhecer e manejar situaçôes de recusa e tomar frente a falsas contraindicaçóes. $\mathrm{Na}$ oportunidade, foi utilizado um paciente-padrão com um simulador pediátrico de baixa fidelidade e um paciente real que fez de mãe, treinada previamente para o cenário. $\mathrm{O}$ tempo destinado à condução da sessão também foi de 45 minutos. Os cenários foram conduzidos pela equipe de pesquisadores $\nabla$ dois enfermeiros com experiência profissional em simulação, doutores em enfermagem $\otimes$ no centro de simulação da ESEnfC.

O questionário de satisfação e autoconfiança na aprendizagem foi utilizado para medir a satisfação dos estudantes e as suas capacidades para a realização de intervençóes de enfermagem após as experiências e conclusão de simulação (NLN, 2004). É importante destacar que esse questionário foi desenvolvido pela National League for Nursing, e deve ser utilizado em simulaçôes de alta fidelidade. No estudo, foi utilizado a versão da escala Student Satisfaction and Self-Confidence in Learning validada para a língua portuguesa. ${ }^{(10)}$ A validação foi realizada com 103 enfermeiros. Nesse processo, a consistência interna (alfa de Cronbach) apresentou valores de 0,86 fator 1 com 06 itens e 0,77 para fator 2 de 07 itens. $^{(10)}$

$\mathrm{O}$ instrumento utilizado possui duas subescalas: satisfação e autoconfiança, total de 13 itens. Na subescala de satisfação, encontra-se 5 itens de avaliação. Já na escala de autoconfiança, oito itens estão incluídos. Ambas são compostas por uma escala de 
likert de 5 itens, a saber: 1 = discordo fortemente da afirmação, 2 = discordo da afirmação, 3 = indeciso - nem concordo nem discordo da afirmação, $4=$ concordo com a afirmação, 5 = concordo fortemente com a afirmação.

Os dados foram analisados com recurso ao SPSS (Pacote Estatístico para Ciências Sociais) versão 24. Para a caracterização do perfil sociodemográfico, utilizou-se a estatística descritiva. $\mathrm{Na}$ análise do desempenho cognitivo e na análise dos scores de satisfação e autoconfiança na aprendizagem, utilizou-se o teste de Mann - Whitney, para um nível de significância de 5\%. Além disso, realizou-se o teste de Teste de Wilcoxon para análise intragrupos, tendo sido adotado o mesmo nível de significância.

\section{Resultados}

Quanto ao perfil sociodemográfico, verificou-se que - dos 94 estudantes - $(95,7 \%)$ são do sexo feminino, com faixa etária entre 18 e 20 anos (91,5\%), solteiros $(98,9 \%)$, sem vínculo laboral $(94,7 \%)$. $\mathrm{Na}$ tabela 1, apresenta-se a caracterização dos pesquisados nos GC e GE.

Tabela 1. Distribuição das frequências e percentuais dos grupos segundo as características sociodemográficas

\begin{tabular}{lccc}
\hline Características & $\begin{array}{c}\text { Grupo } \\
\text { Experimental } \\
\text { sociodemográficas }\end{array}$ & $\begin{array}{c}\text { Grupo Controle } \\
(\mathbf{n}=56)\end{array}$ & $\begin{array}{c}\text { Total } \\
(\mathbf{n}=94)\end{array}$ \\
& $\mathbf{n}(\%)$ & $\mathbf{n}(\%)$ & $\mathbf{n}(\%)$ \\
\hline Faixa etária & & & \\
$\quad$ Entre 18 e 20 anos & $52(92,9)$ & $34(89,4)$ & $86(91,5)$ \\
Entre 21 e 27 anos & $3(5,4)$ & $2(5,2)$ & $5(5,3)$ \\
Entre 28 e 36 anos & $1(1,7)$ & $2(5,2)$ & $3(3,2)$ \\
Sexo & & & \\
Masculino & $1(1,8)$ & $3(7,9)$ & $4(4,3)$ \\
Feminino & $55(98,2)$ & $35(92,1)$ & $90(95,7)$ \\
Estado civil & & & \\
Solteiro & $56(100,0)$ & $37(97,4)$ & $93(98,9)$ \\
Casado & $-(-)$ & $1(2,6)$ & $1(1,1)$ \\
Divorciado & $-(-)$ & $-(-)$ & $-(-)$ \\
União de facto & $-(-)$ & $-(-)$ & $-(-)$ \\
Vínculo laboral & & & \\
Sim & $5(8,9)$ & $-(-)$ & $5(5,3)$ \\
Não & $51(91,1)$ & $38(100,0)$ & $89(94,7)$ \\
\hline
\end{tabular}

No que se refere ao desempenho cognitivo dos pesquisados, a tabela 2 apresenta as scores da avaliação pré (antes da intervenção) e avaliação pós (imediatamente após a intervenção). Conforme exposto, os estudantes do GC apresentaram maior média e menor desvio padrão (DP) na avaliação pré. Em contrapartida, os estudantes do GE atingiram maior média e menor DP na avaliação pós ( $p$-value $=0,000)$.

Tabela 2. Desempenho cognitivo (pré e pós) dos estudantes do grupo controle e experimental e significância estatística

\begin{tabular}{lccccc}
\hline & \multicolumn{2}{c}{$\begin{array}{c}\text { Grupo Controle } \\
(\mathrm{n}=56)\end{array}$} & \multicolumn{2}{c}{$\begin{array}{c}\text { Grupo Experimental } \\
(\mathrm{n}=38)\end{array}$} & p-value \\
& Média & DP & Média & DP & \\
\hline Avaliação Pré & 8,21 & 1,66 & 7,10 & 1,90 & 0,005 \\
Avaliação Pós & 7,84 & 1,70 & 9,50 & 0,87 & 0,000 \\
\hline
\end{tabular}

Além da comparação intergrupos, foi realizado o Teste de Wilcoxon para análise intragrupos. Nesta análise o GE também apresentou maior evolução entre as médias das avaliaçóes pré e pós, obtendo-se significância estatística ( $\mathrm{p}$ valor $=0,000)$. Já os estudantes do GC ficaram estagnados quanto ao aumento dos scores de desempenho cognitivo ( $p$ valor $=0,242)$. Na tabela 3, estão expostas as médias, DP, e o p-value dos GC e GE quanto à satisfação e autoconfiança na aprendizagem. No que se refere à confiabilidade interna, temos que o alpha cronbach total foi de 0,850 . Sendo 0,820 na subescala de satisfação e 0,761 na subescala de autoconfiança.

\section{Discussão}

Nesta pesquisa, os estudantes que participaram da sessão de simulação obtiveram melhores desempenhos - no pós-teste - em relação aos estudantes que participaram da estratégia tradicional. Outros estudos estáo em consonância com os achados deste artigo. ${ }^{(11-14)}$

A simulação clínica potencializa o desempenho cognitivo dos estudantes de enfermagem. Além disso, estudos apontam para a simulação como uma estratégia que permite que a aprendizagem seja significativa. ${ }^{(15)}$ Para que a aprendizagem seja significativa o estudante precisa perceber a aplicabilidade daquilo que está estudando. ${ }^{(16)}$

A participação do estudante, durante o debriefing, potencializa a experiência vivida e traz significado ao que foi experimentado. ${ }^{(17,18)}$ Além disso, o estudado reflete sobre a ação e aprimora o aprendizado para situaçôes futuras. ${ }^{(19)}$ 
Tabela 3. Satisfação dos estudantes e autoconfiança na aprendizagem

\begin{tabular}{|c|c|c|c|c|c|}
\hline \multirow{2}{*}{$\begin{array}{l}\text { Satisfação com a aprendizagem } \\
\text { atual }\end{array}$} & \multicolumn{2}{|c|}{$\begin{array}{l}\text { Grupo Controle } \\
(n=56)\end{array}$} & \multicolumn{3}{|c|}{ Grupo Experimental $(n=38)$} \\
\hline & Média & DP & Média & DP & $p$-value \\
\hline $\begin{array}{l}\text { 1. Os métodos de ensino utilizados } \\
\text { nesta simulação foram úteis e } \\
\text { eficazes. }\end{array}$ & 4,28 & 0,51 & 4,80 & 0,40 & 0,000 \\
\hline $\begin{array}{l}\text { 2. A simulação forneceu-me uma } \\
\text { variedade de materiais didáticos } \\
\text { e atividades para promover a } \\
\text { minha aprendizagem do currículo } \\
\text { de tópicos de Atenção Primária } \\
\text { (imunização de adultos). }\end{array}$ & 4,00 & 0,69 & 4,41 & 0,80 & 0,003 \\
\hline $\begin{array}{l}\text { 3. Eu gostei do modo como o } \\
\text { meu professor ensinou através da } \\
\text { simulação. }\end{array}$ & 4,55 & 0,55 & 4,82 & 0,43 & 0,006 \\
\hline $\begin{array}{l}\text { 4. Os materiais didáticos utilizados } \\
\text { nesta simulação foram motivadores } \\
\text { e ajudaram-me a aprender. }\end{array}$ & 4,18 & 0,65 & 4,57 & 0,59 & 0,003 \\
\hline $\begin{array}{l}\text { 5. A forma como o meu professor } \\
\text { ensinou através da simulação foi } \\
\text { adequada para a forma como eu } \\
\text { aprendo. }\end{array}$ & 4,36 & 0,63 & 4,76 & 0,42 & 0,001 \\
\hline $\begin{array}{l}\text { A autoconfiança na } \\
\text { aprendizagem }\end{array}$ & & & & & \\
\hline $\begin{array}{l}\text { 6. Estou confiante de que domino } \\
\text { o conteúdo da atividade de } \\
\text { simulação que o meu professor me } \\
\text { apresentou. }\end{array}$ & 3,50 & 0,76 & 3,51 & 0,66 & 0,620 \\
\hline $\begin{array}{l}\text { 7. Estou confiante que esta } \\
\text { simulação incluiu o conteúdo } \\
\text { necessário para o domínio do } \\
\text { currículo de tópicos de Atenção } \\
\text { Primária (imunização de adultos). }\end{array}$ & 3,89 & 0,76 & 4,16 & 0,73 & 0,118 \\
\hline $\begin{array}{l}\text { 8. Estou confiante de que estou } \\
\text { desenvolvendo habilidades e } \\
\text { obtendo os conhecimentos } \\
\text { necessários a partir desta } \\
\text { simulação para executar os } \\
\text { procedimentos necessários em um } \\
\text { ambiente clínico. }\end{array}$ & 4,13 & 0,62 & 4,23 & 0,60 & 0,386 \\
\hline $\begin{array}{l}\text { 9. } 0 \text { meu professor utilizou recursos } \\
\text { úteis para ensinar a simulação. }\end{array}$ & 4,44 & 0,55 & 4,66 & 0,51 & 0,051 \\
\hline $\begin{array}{l}\text { 10. É minha responsabilidade como } \\
\text { aluno aprender o que eu preciso } \\
\text { saber através da atividade de } \\
\text { simulação. }\end{array}$ & 4,42 & 0,59 & 4,60 & 0,49 & 0,146 \\
\hline $\begin{array}{l}\text { 11. Eu sei como obter ajuda quando } \\
\text { eu não entender os conceitos } \\
\text { abordados na simulação. }\end{array}$ & 4,13 & 0,57 & 4,44 & 0,63 & 0,011 \\
\hline $\begin{array}{l}\text { 12. Eu sei como usar atividades } \\
\text { de simulação para aprender } \\
\text { habilidades. }\end{array}$ & 3,86 & 0,62 & 4,12 & 0,66 & 0,062 \\
\hline $\begin{array}{l}\text { 13. É responsabilidade do professor } \\
\text { dizer-me o que eu preciso aprender } \\
\text { na temática desenvolvida na } \\
\text { simulação durante a aula. }\end{array}$ & 3,71 & 0,89 & 3,33 & 0,87 & 0,058 \\
\hline
\end{tabular}

Por viabilizar esclarecimentos estruturados e a participação ativa do estudante no processo de ensino e aprendizagem, a simulação - a partir do debriefing - apresenta-se como uma estratégia de maior impacto para o estudante quando comparada a estratégias mais tradicionais. ${ }^{(20)}$

No que se refere a satisfação com a aprendizagem atual e a autoconfiança na aprendizagem pode obser- var-se que, em ambas as subescalas, os estudantes que participaram do GE apresentaram maiores médias e menores DP. Na primeira subescala, houve significância estatística nas cinco variáveis que a compóe.

Um estudo com 199 estudantes do curso de graduação em enfermagem que examinou a percepção sobre a sua satisfação e autoconfiança na simulação concluiu que os pesquisados ficaram satisfeitos com a experiência e sentiram-se confiantes no seu desempenho. (21) Outro estudo, Saudita, com 117 estudantes de enfermagem também mostrou que a simulação como estratégia para a educação clínica promove a satisfação dos alunos com a aprendizagem e melhora sua autoconfiança. ${ }^{(22)}$

Quanto à subescala da autoconfiança na aprendizagem, os estudantes do GE também apresentaram maiores médias e menores DP. Entretanto, só houve significância estatística de forte associação na variável 11 ( $\mathrm{p}$ valor=0,011).

Ainda na subescala de autoconfiança na aprendizagem, destaca-se a variável 13 que trata do professor enquanto figura central no processo de ensino e aprendizagem. Na variável, os estudantes do GC apresentam média de 3,71, DP de 0,89. Já os estudantes do GE obtiveram valores inferiores (média de 3,33 e DP de 0,87).

Os resultados deste estudo também estão em consonância com outras pesquisas no que se refere à simulação enquanto estratégia geradora de satisfação

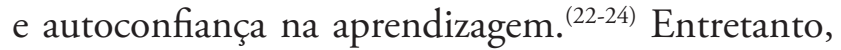
as estratégias tradicionais de ensino também podem gerar esses sentimentos. ${ }^{(1)}$

Em um estudo português, os estudantes referiram que o debriefing estruturado permitiu aumentar a autoconfiança, desenvolver competências de liderança, aumentar o potencial de trabalho em equipa, desenvolver a relação de ajuda, promover a autoconsciência, identificar dificuldades nas suas atuaçôes, assim como melhorar a capacidade de gerir emoções. ${ }^{(20)}$

Estudantes motivados e satisfeitos aprendem mais e melhor. Altos scores de satisfação com a prática simulada pode ser um estímulo para que as escolas de enfermagem invistam nessa estratégia. (7) Além disso, identificar a satisfação dos estudantes pode servir de avaliação do alcance dos objetivos de aprendizagem e do próprio desempenho desses sujeitos. ${ }^{(2,25)}$ 
Nessa perspectiva, a simulação clínica precisa ser pensada dentro do currículo de enfermagem. Definir os objetivos de aprendizagem parece ser o passo inicial para se pensar nas estratégias de ensino e aprendizagem que serão adotadas durante a formação. Quando se pensa em vivenciar e ou treinar condutas mais complexas, a simulação ganha destaque em relação a estratégias mais tradicionais uma vez que permite uma aproximação com a realidade. ${ }^{(4)}$

Além da aproximação com a realidade, estudos mostram vantagens dessa estratégia em detrimento de outras no ensino de enfermagem, a saber: vivenciar situaçóes clínicas independente do acaso; segurança do paciente e do estudante; a utilização prévia de tecnologias disponíveis nos equipamentos de saúde, entre outras. ${ }^{(22,25)}$ Investigações na área da simulação clínica também tem contribuído para o desenvolvimento de diversas competências e habilidades específicas e transversais. ${ }^{(26)}$

\section{Conclusão}

O estudo evidenciou que, a partir da vivência na simulação clínica como estratégia de ensino e aprendizagem para o ensino de consulta de enfermagem em vacinação no ensino de enfermagem comunitária, os estudantes aprendem mais quando comparado aos estudantes que participaram da estratégia tradicional - aula de habilidades ( $\mathrm{p}$ valor $=0,000$ ). Portanto, no contexto estudado, a simulação clínica mostrou-se uma estratégia mais eficaz em relação a aula de habilidades. Além disso, a simulação clínica é uma estratégia que promove satisfação entre os estudantes. Nos cinco itens da subescala de satisfação com a aprendizagem atual foi possível identificar significância estatística, o que reforça o caráter potencial da simulação enquanto estratégia de ensino e aprendizagem. No que se refere à autoconfiança na aprendizagem, os estudantes do GE apresentaram maiores médias e menores DP. Como limitaçóes, aponta-se as perdas - no total de 26 estudantes- em razão do preenchimento parcial de algum dos instrumentos da pesquisa. Além disso, o tempo de seguimento foi relativamente curto. Uma outra limitação foi o pequeno número de estudos que comparam as estratégias mais tradicionais e a simulação no ensino de consulta de enfermagem em vacinação no contexto da enfermagem comunitária. Nessa perspectiva, não foi possível realizar um diálogo mais profundo, comparativo, das evidências extraídas e outros estudos já concluídos. Espera-se que o estudo contribua para responder a lacunas na literatura quanto à eficácia da simulação, do ponto de vista do desempenho cognitivo, em diferentes contextos do ensino de enfermagem. Sugere-se a realização de novos estudos, de cunho experimental e quase-experimentos, para que se possa reforçar as evidências científicas.

\section{Agradecimentos}

Os autores agradecem a Coordenação de Aperfeiçoamento do Pessoal de Nível Superior (CAPES) a bolsa de doutorado sanduíche para Costa RRO e por seu apoio no desenvolvimento da pesquisa; e ao Conselho Nacional de Ciência e Tecnologia (CNPq) a bolsa de produtividade em pesquisa nível PQ2 para Medeiros SM.

\section{Colaborações}

Costa RRO, Medeiros SM, Coutinho VRR contribuíram com a concepção do projeto, análises e interpretação dos dados, redação do artigo, revisão crítica relevante do conteúdo intelectual e aprovação final da versão a ser publicada. Veríssimo CMF, Silva MANCGMM, e Lucena EES contribuíram nas análises e interpretação dos dados, redação do artigo, revisão crítica relevante do conteúdo intelectual e aprovação final da versão a ser publicada.

\section{Referências}

1. Oliveira Costa RR, Medeiros SM, Amado Martins JC, Dias Coutinho VR, A simulação no ensino de enfermagem: reflexões e justificativas a luz da bioética e dos direitos humanos. Acta Bioeth. 2018;24(1):31-8.

2. Meska MH, Franzon JC, Cotta Filho CK, Pereira G Junior, Mazzo A. Satisfaction and self-confidence of nursing students in simulated scenarios with the use of unpleasant odors: randomized clinical trial. Sci Med (Phila). 2018;28(1):ID28693. 
3. Oliveira Costa RR, Medeiros SM, Amado Martins JC, Enders BC, Lira AL, Araújo MS. A Simulação no ensino de enfermagem: uma análise conceitual. Rev Enferm Centro-Oeste Mineiro. 2018;8:e1928.

4. Jeffries PR. Simulation in nursing education: From conceptualization to evaluation. New York: National League for Nursing; 2012.

5. Miller GE. The assessment of clinical skills/competence/performance. Acad Med. 1990;65(9 Suppl):S63-7.

6. Meska MH, Mazzo A, Jorge BM, Souza-Junior VD, Negri EC, Chayamiti EM. Urinary retention: implications of low-fidelity simulation training on the self-confidence of nurses. Rev Esc Enferm USP. 2016;50(5):831-7.

7. Baptista RC, Martins JC, Pereira MF, Mazzo A. Students' satisfaction with simulated clinical experiences: validation of an assessment scale. Rev Lat Am Enfermagem. 2014;22(5):709-15.

8. Grey M. Desenhos experimentais e quase-experimentais. In: LobiondoWood G, Haber J. Pesquisa em enfermagem métodos, avaliação crítica e utilização. 4a ed. Rio de Janeiro: Guanabara-Koogan; 2001. p. 98-109.

9. National League for Nursing (NLN). Simulation Innovation Resource Center [Internet]. Washington, DC: NLN; 2013 [cited 2018 Nov 10]. Avaialble from: http://sirc.nln.org/mod/glossary/view.php?id=183

10. Almeida RG, Mazzo A, Martins JC, Baptista RC, Girão FB, Mendes IA. Validação para a língua portuguesa da escala Student Satisfactionand SelfConfidence in Learning. Rev Lat Am Enfermagem. 2015;23(6):1007-13.

11. Costa RR. Eficácia da simulação realística no ensino de imunização de adultos no contexto da graduação em enfermagem [tese]. Natal: Universidade Federal do Rio Grande do Norte; 2018.

12. Miranda RP, de Cássia Lopes Chaves É, Silva Lima R, Braga CG, Simões $I A$, Fava SM, et al. The effectiveness of a simulated scenario to teach nursing students how to perform a bed bath: A randomized clinical trial. Nurse Educ Today. 2017;57:17-23.

13. Akhu-Zaheya LM, Gharaibeh MK,AlostazZM. Effectiveness of simulation on knowledge acquisition, knowledge retention, and self-efficacy of nursing students in Jordan. Clin Simul Nurs. 2013;9(9):e335-42.

14. Merriman CD, Stayt LC, Ricketts B. Comparing the Effectiveness of Clinical Simulation versus Didactic Methods to Teach Undergraduate Adult Nursing Students to Recognize and Assess the Deteriorating Patient. Clin Simul Nurs. 2014;10(3):e119-e127.
15. Leigh GT. High-fidelity patient simulation and nursing students' self-efficacy: a review of the literature. Int J Nurs Educ Scholarsh. 2008;5(1):article 37.

16. Oliveira SN, Prado ML, Kempfer SS, Waterkemper R, Morera JA, Bernardi MC. Learning through clinical experience simulation: perceptions in nursing students. Rev Iberoam Educ Invest Enferm. 2015;5(3):56-63.

17. Kolbe M, Grande B, Spahn DR. Briefing and debriefing during simulation-based training and beyond: Content, structure, attitude and setting. Best Pract Res Clin Anaesthesiol. 2015;29(1):87-96.

18. Gardner R. Introduction to debriefing. Semin Perinatol. 2013;37(3):16674.

19. Martins JC. Learning and development in simulated practice environments. Enferm Ref. 2017;4(12):155-62.

20. Coutinho VR, Martins JC, Pereira F. Student's opinion about feedback VS debriefing: a randomized controlled trial. Internacional J Nurs Didatics. 2017;7(4):63-8.

21. Zapko KA, Ferranto ML, Blasiman R, Shelestak D. Evaluating best educational practices, student satisfaction, and self-confidence in simulation: A descriptive study. Nurse Educ Today. 2018;60:2834.

22. Omer T. Nursing students' perceptions of satisfaction and selfconfidence with clinical simulation experience. J Educ Pract. 2016;7(5):131-8.

23. Swenty CF, Eggleston BM. The Evaluation of Simulation in a Baccalaureate Nursing Program. Clin Simul Nurs. 2011;7(5):181-7.

24. Xiaoying MA. BSN Students' perception of satisfaction and selfconfidence after a simulated mock code experience: a descriptive study [thesis]. Cedarville University; 2013.

25. Sigalit W, Sivia B, Michal I. Factors associated with nursing students' resilience: communication skills course, use of social media and satisfaction with clinical placement. J Prof Nurs. 2017;33(2):15361.

26. Costa RR, Medeiros SM, Martins JC, Cossi MS, Araújo MS. Percepção de estudantes da graduação em enfermagem sobre a simulação realística. Rev Cuid (Bucaramanga). 2017;8(3):1799-808. 